\title{
Orthogonality catastrophe and decoherence in a trapped-Fermion environment
}

\author{
A. Sindona ${ }^{1,2}$, J. Goold ${ }^{3,4}$, N. Lo Gullo ${ }^{4,5}$, S. Lorenzo ${ }^{1,2}$, F. Plastina ${ }^{1,2}$ \\ ${ }^{1}$ Dipartimento di Fisica, Università della Calabria, 87036 Arcavacata di Rende (CS), Italy \\ ${ }^{2}$ INFN sezione LNF-Gruppo collegato di Cosenza, Italy \\ ${ }^{3}$ Clarendon Laboratory, University of Oxford, United Kingdom \\ ${ }^{4}$ Physics Department, University College Cork, Cork, Ireland \\ ${ }^{5}$ Quantum Systems Unit, Okinawa Institute of Science and Technology and Graduate University, Okinawa, Japan
}

(Dated: October 30, 2018)

\begin{abstract}
The Fermi edge singularity and the Anderson orthogonality catastrophe describe the universal physics which occurs when a Fermi sea is locally quenched by the sudden switching of a scattering potential, leading to a brutal disturbance of its ground state. We demonstrate that the effect can be seen in the controllable domain of ultracold trapped gases by providing an analytic description of the out-of-equilibrium response to an atomic impurity, both at zero and at finite temperature. Furthermore, we link the transient behavior of the gas to the decoherence of the impurity, and, in particular to the amount of non-markovianity of its dynamics.
\end{abstract}

PACS numbers: 67.85.-d, 05.30.Fk, 03.65.Yz

A Fermi gas may be shaken-up by the switching of even a single, weakly interacting impurity, producing a complete rearrangement of the many body wave-function that, as a consequence, loses essentially any overlap with the initial, unperturbed one. This is the essence of Anderson's orthogonality catastrophe [1,2], witnessed by the singular (edge-like) behavior of the energy distribution of the impurity induced excitations. An example of how such a many-body effect comes into play is provided by $\mathrm{X}$-ray photoemission spectra from most simple metals, where the expected sharp symmetric peak at the binding energy of a core level is converted into a power law singularity, as predicted by the Mahan-NozièresDominics (MND) theory [3, 4]. Similar patterns have been observed in electron emission via X-ray absorption and Auger neutralization from carbon based nanomaterials [5], and for quantum dots [6]. Fermi edge resonance and orthogonality catastrophe have been also revealed by non-equilibrium current fluctuations (shot noise) in nanoscale conductors [7], and enter prominently the physics of phenomena as diverse as the Kondo effect $[2,8]$ and the scattering or sticking of a lowenergy atom or ion on a metal surface $[9,10]$.

Recently, it has been proposed to observe this universal physics in controllable ultracold atomic setups where the singular behavior may be probed either in the time domain, by Ramsey interference type experiments performed on the impurity atom [11], or in the frequency domain, by radiofrequency spectroscopy [12]. However, an analytic framework for the case of a trapped Fermi gas is lacking. In this letter we provide such an analytic description, and discuss the transient response of a harmonically trapped Fermi gas following the 'sudden' switching of an embedded two-level atom excited by a fast pulse. The interaction with the local impurity produces a local quench of the gas, giving rise to the Anderson catastrophe. We study the Fermi-edge physics at zero and finite temperature and both in the frequency domain, by looking at the excitation spectrum of the gas, and in the time domain, by analyzing the dynamics of the impurity. Thus, we link the Fermi edge behavior of the excitation energy distribution to the decoherence of the impurity. In particular, we investigate the Loschmidt echo $[13,14]$ and the non-Markovianity, using recently developed tools [15-19], employed so far to study open systems in different environments, ranging from spins [20] to Bose-Einstein condensates [21], and experimentally tested in optical set-ups [22, 23]. We find that the nonMarkovianity of the decoherent dynamics of the impurity provides a novel interpretation of the essential physics of the shake-up process.

We consider a gas of non-interacting cold fermions confined by a one-dimensional trapping harmonic potential of frequency $\omega$, and described by the Hamiltonian $\hat{H}_{0}=$ $\sum_{n, \xi} \varepsilon_{n} \hat{c}_{n \xi}^{\dagger} \hat{c}_{n \xi}$, with $\hat{c}_{n \xi}$ being the annihilation operator for the $n$-th single particle state of energy $\varepsilon_{n}=\hbar \omega(n+1 / 2)$ and spin $\xi$. We add a two-level impurity (an atom of a different species from the trapped component), with internal states $|g\rangle$ and $|e\rangle$ and Hamiltonian $\hat{H}_{\mathrm{I}}=\sum_{i=e, g} \epsilon_{i}|i\rangle\langle i|$, which is trapped in an auxiliary potential and brought in contact with the Fermi gas. Such a situation can be achieved using a species selective dipole potential that has a frequency much greater than the trap which contains the gas, so that the impurity motion is essentially frozen. We assume that when the impurity is in the $|g\rangle$ state, it has a negligible scattering interaction with the gas, hence the Hamiltonian of the composite system is given by $\hat{H}=\hat{H}_{0}+\hat{H}_{\mathrm{I}}+\hat{V} \otimes|e\rangle\langle e|$. With the fermions in their equilibrium configuration, set by $\hat{H}_{0}$, we suppose the impurity to be quickly excited so that the gas feels a sudden perturbation $\hat{V}(t)=\hat{V} \theta(t)$ due to the interaction, assumed to have an $s$-wave like character.

As it is standard in ultracold atoms, at sufficiently low temperatures, the pseudo-potential approximation for the interaction is invoked, which amounts to replacing the complicated atomic interaction potential with an effective short range potential of strength $V_{0}$, localized at the minimum of the harmonic well, which we scale with the trap length $x_{0}$ such that $V(x)=\pi V_{0} x_{0} \delta(x)$. Due to the parity of the single particle wave-functions, only the fermions lying in even-parity states $(n=2 r$, with $r=0,1,2, \cdots)$ feel the impurity and are involved in the shake-up process. Explicitly, the fermion-impurity interaction is given by $\hat{V}=$ $\sum_{r, r^{\prime}, \xi} V_{r r^{\prime}} \hat{c}_{2 r \xi}^{\dagger} \hat{c}_{2 r^{\prime} \xi}$, where $V_{r r^{\prime}}=V_{0}(-1)^{r+r^{\prime}} \gamma_{r}^{1 / 2} \gamma_{r^{\prime}}^{1 / 2}$, 
and $\gamma_{r}=2^{-2 r} \pi^{1 / 2}(2 r) ! / r !^{2}$ (See appendix A). We label the highest occupied level by $n_{F}=2 r_{\mathrm{F}}$, with $r_{\mathrm{F}}$ a positive integer, so that the Fermi energy reads $\varepsilon_{\mathrm{F}}=\hbar \omega\left(2 r_{\mathrm{F}}+1 / 2\right)$.

A key quantity for the following is the vacuum persistence amplitude

$$
\nu_{\beta}(t>0)=\left\langle e^{\frac{i}{\hbar} \hat{H}_{0} t} e^{-\frac{i}{\hbar}\left(\hat{H}_{0}+\hat{V}\right) t}\right\rangle,
$$

with $\langle\cdots\rangle$ denoting the grand canonical average over the unperturbed fermion state. $\nu_{\beta}(t)$ is the probability amplitude that the gas will retrieve its equilibrium state at time $t$, after the switching on of the perturbation and, as discussed below, its modulus gives the decoherence factor for the impurity.

The Fourier transform $\tilde{\nu}_{\beta}(E)$, subject to the constraint $\nu_{\beta}(t<0)=\nu_{\beta}^{*}(-t)$, gives the excitation spectrum of the gas. Turning to the interaction picture, we get

$$
\nu_{\beta}(t)=\left\langle T e^{\frac{1}{i \hbar} \int_{0}^{t} d t^{\prime} \tilde{V}\left(t^{\prime}\right)}\right\rangle, \quad \tilde{V}(t)=e^{\frac{i}{\hbar} \hat{H}_{0} t} \hat{V} e^{-\frac{i}{\hbar} \hat{H}_{0} t},
$$

which, by virtue of the linked cluster theorem, reduces to an exponential sum of connected Feynman diagrams, $\nu_{\beta}(t)=$ $e^{\Lambda_{\beta}(t)}$, with:

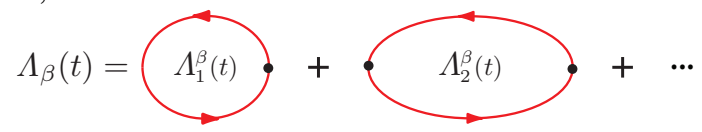

The closed graphs in $\Lambda_{\beta}(t)$ contain products of vertices $\left(V_{r r^{\prime}}\right)$ connected by lines $\left(G_{r}^{\beta}\right)$ that represent the unperturbed propagators

$$
i \hbar G_{r}^{\beta}(t)=e^{-i \varepsilon_{2 r} t / \hbar}\left[\theta(t) f_{r}^{-}-\theta(-t) f_{r}^{+}\right],
$$

where $f_{r}^{ \pm}=\left[1+e^{ \pm \beta\left(\varepsilon_{2 r}-\mu\right)}\right]^{-1}$ are the particle-hole distributions, and $\mu$ denotes the chemical potential (see Appendix C).

We focus on the lowest order loops, namely

$$
\hbar \Lambda_{1}^{\beta}(t)=-i t \chi_{s} V_{0} \lambda_{+}^{\beta}(0),
$$

and

$$
\hbar^{2} \Lambda_{2}^{\beta}(t)=-\chi_{s} V_{0}^{2} \int_{0}^{t} d t^{\prime} \int_{0}^{t^{\prime}} d t^{\prime \prime} \lambda_{+}^{\beta}\left(t^{\prime \prime}\right) \lambda_{-}^{\beta}\left(t^{\prime \prime}\right),
$$

with $\chi_{s}=(2 s+1)$ accounting for the spin degeneracy and $\lambda_{ \pm}^{\beta}(t)=\sum_{r=0}^{\infty} \gamma_{r} e^{ \pm 2 i r \omega t} f_{r}^{ \pm}$.

This approximation will prove to accurately describe the singular response of the gas (contained in the two-vertex term) and to give the dominant contribution to the shake-up process if the interaction strength is small in the energy scale of the problem. The latter is set by both the level separation $\hbar \omega$ and Fermi energy $\varepsilon_{\mathrm{F}}$, which allow us to introduce $\alpha=\frac{\chi_{s} V_{0}^{2}}{2 \hbar \omega \varepsilon_{\mathrm{F}}}$ as a sensible interaction strength parameter.

The contribution (4) may be written as $\hbar \Lambda_{1}^{\beta}(t)=-i t E_{1}^{\beta}$. Here,

$$
E_{1}^{\beta}=\sqrt{2 \chi_{s} \hbar \omega \varepsilon_{\mathrm{F}} \alpha} \sum_{r=0}^{\infty} \gamma_{r} f_{r}^{+}
$$

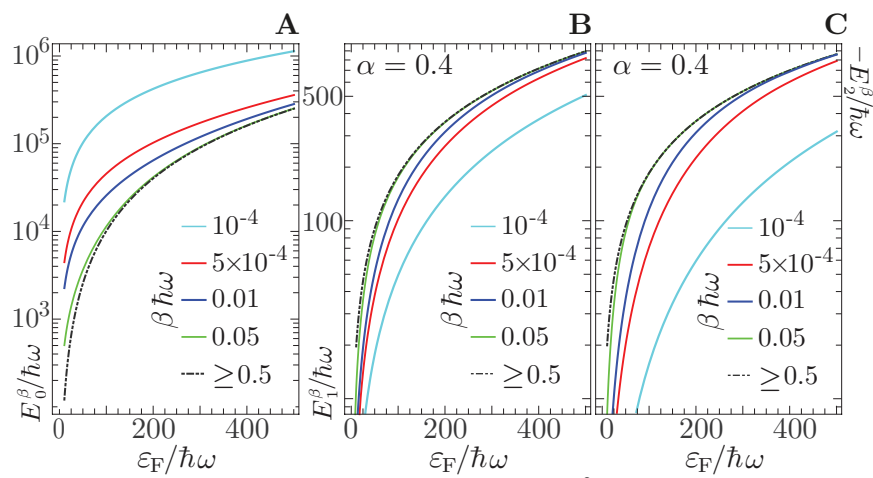

FIG. 1. (Color online) Equilibrium energy $E_{0}^{\beta}$ of a spin-1/2 gas into a harmonic trap (panel A) together with the perturbation corrections $E_{1}^{\beta}$ (Eq. (6), panel B) and $E_{2}^{\beta}$ (Eq. (7), panel C) due to the effective impurity potential $V(x)$. All Energy curves are reported in units of $\hbar \omega v s \varepsilon_{\mathrm{F}} / \hbar \omega$ for different values of $\beta \hbar \omega$ and fixed coupling parameter $\alpha=0.4$.

is the first-order shift to the gas energy, as provided by the Rayleigh-Schrödinger perturbation theory. The behavior of the unperturbed energy $E_{0}^{\beta}=\chi_{s} \sum_{n} \varepsilon_{n} f_{n / 2}^{+}$, and of its first and second order corrections (the latter obtained from the two vertex term, see below) vs $\varepsilon_{\mathrm{F}}$ is shown in Fig. 1 for various temperatures. We notice that $E_{0}^{\beta}$ is 1 to 3 orders of magnitude larger than $E_{1}^{\beta}$ for $\alpha \lesssim 1$, while temperature plays an appreciable role in both $E_{0}^{\beta}$ and $E_{1}^{\beta}$ for $\beta \hbar \omega$ less than $\sim 0.05$.

While $\Lambda_{1}^{\beta}(t)$ only brings a phase factor to $\nu_{\beta}(t)$, which corresponds to shifting the spectrum $\tilde{\nu}_{\beta}(E)$ by $E_{1}^{\beta}$, the twovertex connected graph gives the crucial contribution to the persistence amplitude. As detailed in Appendix B, it can be split into three parts with well defined trends and physical meaning, i.e., $\Lambda_{2}^{\beta}(t)=\Lambda_{2 \mathrm{~S}}^{\beta}(t)+\Lambda_{2 \mathrm{G}}^{\beta}(t)+\Lambda_{2 \mathrm{P}}^{\beta}(t)$. These represent a (further) energy shift, a gaussian envelope due to finite temperature effects, and a periodic terms originating from the equal spacing of the unperturbed single-particle states, respectively, and are separately analyzed in Figs. $1 \mathbf{C}, 2 \mathbf{A}$, and $2 \mathbf{B}$.

The first one, $\hbar \Lambda_{2 \mathrm{~S}}^{\beta}(t)=-i t E_{2}^{\beta}$, provides the second-order correction to the energy of the gas (the $n>2$-vertex graphs would complete the perturbation series):

$$
E_{2}^{\beta}=\alpha \varepsilon_{\mathrm{F}} \sum_{r \neq r^{\prime}=0}^{\infty} \frac{f_{r}^{+} \gamma_{r} \gamma_{r^{\prime}} f_{r^{\prime}}^{-}}{r-r^{\prime}} .
$$

Comparing Fig. 1 $\mathbf{B}$ and $\mathbf{C}$, we notice that the chosen value of $\alpha$ let $E_{2}^{\beta}$ take absolute values smaller than $E_{1}^{\beta}$. However, $E_{2}^{\beta}$ is more sensitive to temperature than $E_{1}^{\beta}$ for $\beta \hbar \omega<0.05$.

The second contribution, $\Lambda_{2 \mathrm{G}}^{\beta}(t)=-\delta_{\beta}^{2} \omega^{2} t^{2} / 2$, produces a Gaussian damping in $\nu_{\beta}(t)$ and, therefore, a Gaussian broadening in $\tilde{\nu}_{\beta}(E)$ with standard deviation

$$
\delta_{\beta}=\sqrt{2 \alpha g_{\beta}}, \quad g_{\beta}=\frac{\varepsilon_{\mathrm{F}}}{\hbar \omega} \sum_{r=0}^{\infty} \gamma_{r}^{2} f_{r}^{+} f_{r}^{-} .
$$

Here, the coefficient $g_{\beta}$ is weakly influenced by the Fermi energy, but strongly affected by temperature, changing by various orders of magnitude for $\beta \hbar \omega \lesssim 0.5$. No damp- 
ing/broadening effects are present at the absolute zero, since $\delta_{\beta} \rightarrow 0$ for $\beta \hbar \omega \rightarrow \infty$ (Fig.2A).

The most important content of the second diagram, giving a non trivial structure to $\nu_{\beta}(t)$, arises from the third contribution (see the Appendix):

$$
\Lambda_{2 \mathrm{P}}^{\beta}(t)=-\frac{\alpha \varepsilon_{\mathrm{F}}}{2 \hbar \omega} \sum_{r \neq r^{\prime}}^{\infty} \gamma_{r} f_{r}^{+} \frac{1-e^{2 i\left(r-r^{\prime}\right) t \omega}}{\left(r-r^{\prime}\right)^{2}} \gamma_{r^{\prime}} f_{r^{\prime}}^{-} .
$$

Due to the harmonic form of trapping potential, this is a periodic function of time with frequency $2 \omega$, see Fig. $2 \mathbf{B}$. The zeroes of this sub-graph (at $\omega t=m \pi$ with $m=0, \pm 1, \pm 2$ ), when combined with the Gaussian damping (8), yield modulations in the vacuum persistence amplitude which, as discussed below, are a signature of non-markovian dynamics of the impurity.

Leaving aside the shifts, the persistence amplitude is then:

$$
\nu_{\beta}^{\prime}(t)=e^{-\delta_{\beta} \omega^{2} t^{2} / 2} e^{\Lambda_{2 \mathrm{P}}^{\beta}(t)} .
$$

Of particular interest for the discussion below is the behavior of $\left|\nu_{\beta}(t)\right|$ exhibiting spikes at $\omega t \sim \pi, 2 \pi, \cdots$, which become more and more pronounced with increasing $\beta \hbar \omega$, see left panels in Fig. 3. The periodicity in the time domain is reflected in the excitation spectrum $\tilde{\nu}_{\beta}(E)$ that offers an asymmetric, broadened, signature of the singular behavior of the Fermi gas. The monotonic structure turns into a sequence of sub-peaks, separated by $2 \hbar \omega$ and related to even-level transitions in the gas as $\beta \hbar \omega$ gets above $\sim 0.5$ (see Fig. 3B). These features are observed for any $r_{\mathrm{F}}$ in the range of 5 to 100 (see Appendix C).

The coefficient (8) of the Gaussian power law and the periodic contribution $\Lambda_{2 \mathrm{P}}^{\beta}(t)$ can be approximated as

$$
g_{\beta} \approx 2 \sum_{m=1}^{\infty}(-1)^{m} m \frac{e^{\beta \hbar \omega m / 2}}{e^{\beta \hbar \omega m}-1},
$$

and

$$
\Lambda_{2 \mathrm{P}}^{\beta}(t) \approx \alpha \sum_{m=-\infty}^{\infty} \ln \frac{e^{2 \tau_{m} \omega}-1}{e^{2\left(i t+\tau_{m}\right) \omega}-1} .
$$

At low temperatures, the leading behavior of the Gaussian standard deviation is $\delta_{\beta} \approx 2 \alpha^{1 / 2} e^{-\beta \hbar \omega / 4}$ for thermal energies $\beta \hbar \omega \gtrsim 6$ (see Fig. $2 \mathbf{A}$ ). On the other hand, Eq. (12) contains a singularity at the absolute zero, that we regularized by introducing a cut-off parameter $\tau_{0}$. This regularization is only needed to remove a zero temperature indefiniteness of the analytic approximation, whereas the numerical evaluation of the vacuum persistence amplitude does not suffer from divergence problems. As shown below and as detailed in the Appendices below, a similar parameter enters the original MND theory, and we can interpret it as the typical time-scale over which transitions occur in the gas. On the other hand, thermal fluctuations introduce other characteristic times $\tau_{m}=m \beta \hbar$.

Taking $g_{\beta}$ and $\Lambda_{2 \mathrm{P}}^{\beta}(t)$ as in Eqs. (11) and (12), and using them in Eq. (10) gives an accurate approximation to the numerical results for $\beta \hbar \omega \gtrsim 0.1$, number of particles larger
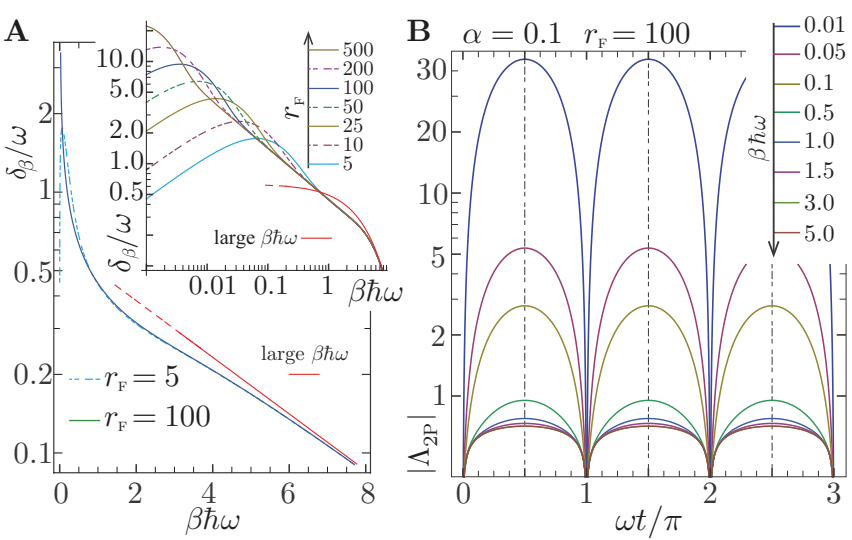

FIG. 2. (color online): (A) Standard deviation in the Gaussian power law (8), expressed in $\omega$-units, $v s \beta \hbar \omega$ for $r_{\mathrm{F}}=5-100$ and $\alpha=0.1$. The low thermal energy approximation introduced in the text is also reported. (B) Periodic component $\Lambda_{2 \mathrm{P}}^{\beta}(t) v s \omega t / \pi$, for $r_{\mathrm{F}}=100$, $\beta \hbar \omega=0.01-5$ and $\alpha=0.1$

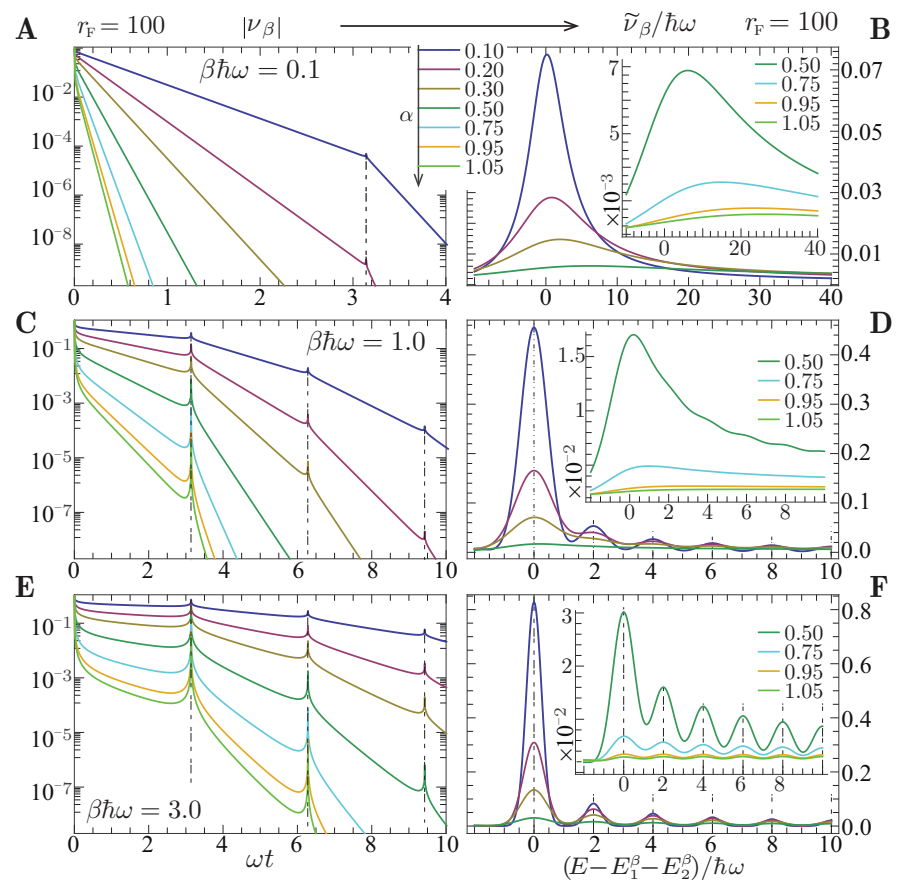

FIG. 3. (color online): Absolute value of the decoherence factor $\left|\nu_{\beta}(t)\right|$ (left panels, A,C,E) and excitation spectrum $\nu_{\beta}^{\prime}(E)$ (right panels, B,D,F), calculated from eq. (10) by numerically computing the Gaussian damping (8) and the periodic sub-diagram $\Lambda_{2 \mathrm{p}}^{\beta}(t)$, for $\beta \hbar \omega=0.1-3.0, r_{\mathrm{F}}=100$, and $\alpha=0.1-1.05$.

than 10 and for suitable values of the cut-off parameter, say, $\omega \tau_{0}<0.02$ (see Fig. 4A). In particular, at $T=0$, the vacuum persistence amplitude takes the form:

$$
\nu_{\beta \rightarrow \infty}^{\prime}(t) \approx\left[\frac{e^{2 \tau_{0} \omega}-1}{e^{2 \omega\left(\tau_{0}+i t\right)}-1}\right]^{\alpha} .
$$

To compare our findings to the one-dimensional freefermion theory, one needs to fix $\alpha$ and let the harmonic frequency go to zero by keeping the number of particles in the gas $\left(2 r_{\mathrm{F}} \approx \varepsilon_{\mathrm{F}} / \hbar \omega\right)$ finite. No Gaussian damping occurs in this 
case, and the two vertex graph tends to

$$
\Lambda_{\mathrm{MND}}(t)=-\alpha \ln \left(i t / \tau_{0}+1\right) \text {, }
$$

yielding the Nozieres-De Dominicis propagator $\nu_{\mathrm{MND}}(t)=$ $\left(i t / \tau_{0}+1\right)^{-\alpha}$, originally calculated for a suddenly switched on core-hole in a free electron gas [4]. Eq. (14) was obtained by writing down a generalized Dyson equation for the electron Green's function in a constant window potential of width $\hbar / \tau_{0}$, and solving it for all connected graphs in the long-time limit. For this reason, the MND spectrum lacks formal justification away from the threshold. In the present derivation, we have taken into account the full perturbation at an arbitrary time $t>0$, retaining only the first non adiabatic contribution in the linked cluster expansion [24]. We expect the effect of higher order diagrams to be mainly concerned with the adiabatic correction to the equilibrium energy and some additional broadening of the excitation peaks. The latter should provide a renormalization to the critical parameter. Nevertheless, in the investigated ranges of temperatures and particle numbers, the definition of $\alpha$ given here produces a markedly singular response with the same range of criticality as the MND edge response parameter $(\alpha=0-1)$.

From this comparison with the free-gas case, we learn that the trapping frequency $\omega$ enters crucially the physics of the shake up process. Indeed, it modifies the long time response of the gas as all single particle excitations involve energy exchanges which are now even multiples of $\hbar \omega$. This gives rise to the periodic part of the fermion response and to the corresponding spectral peaks which are then broadened at finite temperatures due to the gaussian envelope, the latter being a typical effect of suddenly switched perturbations [2]. Up
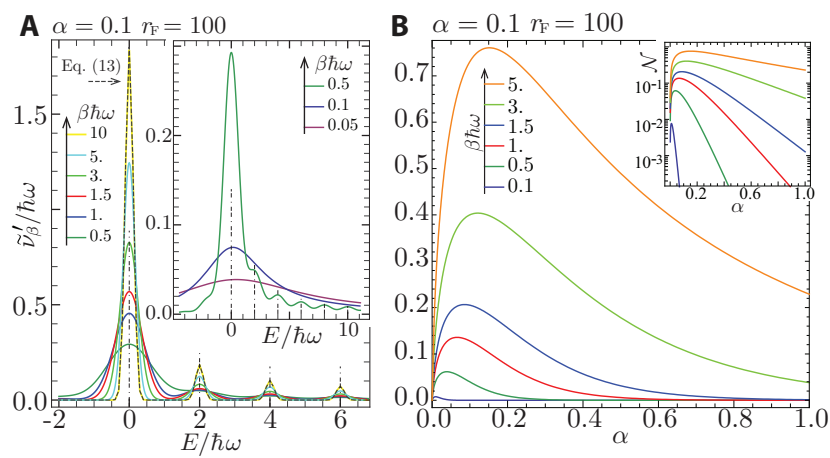

FIG. 4. (color online): (A) Absorption spectrum $\nu_{\beta}^{\prime}(E)$, calculated numerically from eq. (10) with $\beta \hbar \omega=0.1-\infty, r_{\mathrm{F}}=100$, and $\alpha=0.1$, and analytical approximation $\nu_{\beta}^{\prime}(E)$ obtained from Eqs. (11) and (12) with $\hbar \omega \beta=10$ and $\omega \tau_{0}=0.001$; (B) NonMarkovianity measure as a function of the critical parameter $\alpha$ for various temperatures.

to now, we treated the response of the Fermi gas without any reference to the dynamics of the impurity, that has just been assumed in the excited states for $t \geq 0$. If, instead, the two-level atom is subject to (say) a fast $\pi / 2$ pulse and quickly prepared in the superposition $(|g\rangle+|e\rangle) / \sqrt{2}$, it experiences a purely dephasing dynamics due to the coupling with the gas, such that its state at later times is $\rho_{\mathrm{IMP}}(t)=$ $\left(|g\rangle\langle g|+| e\rangle\left\langle e\left|+\nu_{\beta}(t)\right| g\right\rangle\langle e|+\right.$ h.c. $) / 2$. The decoherence factor that enters the off-diagonal elements is just the persistence amplitude that we obtained before, going to zero at long times due to the orthogonality catastrophe. In the theory of open systems, one typically uses a related function, the so called Loschmidt echo $L(t)=\left|\nu_{\beta}(t)\right|^{2}$, which gives a measure of the environmental response to the perturbation induced by the system [13, 14, 25] and which, as shown in Ref. [19], is linked with non-Markovianity of the open system dynamics. The amount of non-Markovianity of a dynamical map can be evaluated in different manners [15-18], which are, however, essentially equivalent for a purely dephasing quantum channel $[19,26]$. By adopting the definition in terms of information flow given in [15], one finds

$$
\mathcal{N}=\sum_{n}\left\{\left|\nu_{\beta}\left(t_{\max , n}\right)\right|-\left|\nu_{\beta}\left(t_{\min , n}\right)\right|\right\},
$$

where the sum is performed over all maxima and minima of the $\left|\nu_{\beta}(t)\right|$, occurring at $t_{\max , n}$ and $t_{\min , n}$, respectively. Using our previous results for the amplitude, we can then obtain the non-Markovianity of the dynamics of a two-level system in a trapped Fermi environment. The results are shown in Fig. 4B, where we see that $\mathcal{N}$ depends on the temperature and on the critical parameter $\alpha$. In particular, it has a maximum at small $\alpha$, increasing with low temperatures, and goes to zero both for large temperatures (due to the fact that thermal fluctuations suppress oscillations in the persistence amplitude) and for $\alpha>1$. In the latter case, excitations are generated at every energy scale in the fermion gas, as witnessed by the fact that the spectrum becomes structure-less. This implies that the gas becomes more and more stiff (in the sense that it is not able to react on the impurity any more) and explains why $\mathcal{N}$ is zero: the open system does not receive information back, its Loschmidt echo decays monotonously and thus the dynamics is Markovian. As a result, we conclude that a non-Markovian dynamics can be characterized, in our case, by the appearance of specific spectral features in the excitation energy distribution [27].

We conclude with two remarks. First, the spectral distribution of energy excitations obtained in the present work coincides with the so called work distribution function, which is a central quantity in non-equilibrium processes [28]. In the set-up that we have described above, it is simple enough to conceive a 'reverse' protocol, with the fermi gas brought to thermal equilibrium in the presence of the impurity (i.e. with the two-level atom in the excited state) which is then switched off. The comparison of the work distribution functions in the direct and reverse protocols would lead to a direct experimental test of the Crooks relation in the quantum regime [29]. The second remark is on the experimental realization of the model that we have described. Many experiments have recently dealt with the effects of impurities in trapped Fermi gas [30], and state-dependent scattering lengths have been discussed [31]. This would lead to a direct test of our theory. Another viable candidate could be a gas of hard-core bosons in onedimension, where the Loschmidt echo is equivalent to that of the corresponding Fermi gas [32] and in which impurities have recently been experimentally generated [33]. 
[1] P. W. Anderson, Phys. Rev. Lett. 18, 1049 (1967).

[2] P. W. Anderson and G. Yuval, Phys. Rev. Lett. 23, 89 (1969); P. W. Anderson, G. Yuval and R. D. Hamann, Phys. Rev. B 1, 4464 (1970).

[3] G.D. Mahan, Phys. Rev. 163, 612 (1967).

[4] P. Nozières and C.T. De Dominicis, Phys.Rev. 178, 1097 (1969); R. Roulet, J. Gavoret and P. Nozières, Phys.Rev. 178, 1072 (1969); P. Nozieres, J. Gavoret and R. Roulet, Phys.Rev. 178, 1084 (1969).

[5] A. Sindona et al., Surf. Sci., 601, 2805 (2007); A. Sindona, M. Pisarra, P. Riccardi, G. Falcone, Nanosci. Nanotechnol. Lett. 4, 1050 (2012). A. Sindona et al., J. Phys.: Condens. Matter 25 115301 (2013)

[6] K. A. Matveev and A. I. Larkin, Phys. Rev. B 46, 15337 (1992); A. K. Geim et al., Phys. Rev. Lett. 72, 2061 (1994); H. Frahm, C. von Zobeltitz, N. Maire, R. J. Haug, Phys. Rev. B 74, 035329 (2006); M. Heyl and S. Kehrein, Phys. Rev. B 85, 155413 (2012).

[7] N. Ubbelohde et al., Sci. Rep. 2:374, 1 (2012).

[8] M. Hentschel and F. Guinea, Phys. Rev. B 76, 115407 (2007).

[9] A. Sindona, R.A. Baragiola, G. Falcone, A. Oliva, P. Riccardi, Phys. Rev. A 71, 052903 (2005)

[10] D. P. Clougherty and Y. Zhang, Phys. Rev. Lett 109, 120401 (2012).

[11] J. Goold, T. Fogarty, N. Lo Gullo, M. Paternostro, and T. Busch, Phys. Rev. A 84, 063632 (2011).

[12] M. Knap et al., Phys. Rev. X 2, 041020 (2012).

[13] A. Peres, Phys. Rev. A 30, 1610 (1984); T. Gorin et al., Phys. Rep. 435, 33 (2006).

[14] F. M. Cucchietti, et al., Phys. Rev. Lett. 91, 210403 (2003).

[15] H. P. Breuer, E.-M. Laine, and J. Piilo, Phys. Rev. Lett. 103, 210401 (2009).

[16] A. Rivas, S. F. Huelga, and M. B. Plenio, Phys. Rev. Lett. 105, 050403 (2010).

[17] X. M. Lu, X. Wang, and C. P. Sun, Phys. Rev. A 82, 042103 (2010).

[18] S. Lorenzo, F. Plastina, M. Paternostro, Phys. Rev. A (R) to be published (2013), arXiv:1302.6673 (2013).

[19] P. Haikka et al., Phys. Rev. A 85, 060101(R) (2012).

[20] T. J. G. Apollaro et al., Phys. Rev. A 83, 032103 (2011); S. Lorenzo, F. Plastina, and M. Paternostro, Phys. Rev. A 87, 022317 (2013).

[21] P. Haikka et al. Phys. Rev. A 84, 031602(R) (2012).

[22] B-H. Lieu et al., Nat. Phys. 7, 931 (2011).

[23] A. Chiuri et al., Scientific Report 2, 968 (2012).

[24] A complementary, bosonization based approach to the response of a free electron gas is provided by K. D. Schotte and U. Schotte, Phys. Rev. 182, 479 (1969).

[25] P. Zanardi and N. Paunkovíc, Phys. Rev. E. 74, 031123 (2006).

[26] H.-S. Zeng et al., Phys. Rev. A 84, 032118 (2011).

[27] Similar conclusions have been reported in W.-M. Zhang et al., Phys. Rev. Lett. 109, 170402 (2012).

[28] M. Campisi, P. Hänggi, and P. Talkner, Rev. Mod. Phys. 83, 771 (2011).

[29] M. Heyl and S. Kehrein, Phys. Rev. Lett. 108, 190601 (2012).

[30] A. Schirotzek et al., Phys. Rev. Lett. 102, 230402 (2009); C. Kohstall et al., Nature 485, 615 (2012); M. Koschorreck et al., Nature 485, 619 (2012).

[31] K. M. Daily, D. Rakshit, and D. Blume, Phys. Rev. Lett. 109, 030401 (2012).

[32] K. Lelas, T. S̆eva, and H. Buljan, Phys. Rev. A 84, 063601
(2011); K. Lelas, T. S̆eva, H. Buljan and J. Goold, Phys. Rev. A 86, 033620 (2012).

[33] S. Palzer, C. Zipkes, C. Sias, and M. Köhl, Phys. Rev. Lett. 103, 150601 (2009); J. Catani et al., Phys. Rev. A 85, 023623 (2012).

\section{Appendix A: Impurity potential}

As explained in the main text, the non-interacting fermions in the harmonic trap lie in their equilibrium configuration, set by $\hat{H}_{0}$, until the impurity is excited and the sudden perturbation $\hat{V}(t)=\hat{V} \theta(t)$ is felt by the gas. To mimic a very strong difference in scattering length depending on the internal state $|e\rangle$ of the impurity, we have modelled it by a spatially localized potential, activated by the population of the excited state, with the structure-less form $V(x)=\pi V_{0} x_{0} \delta(x)$. For mathematical simplicity we have placed the impurity at the minimum of the harmonic potential. Thus, the coupling matrix elements between two unperturbed one-fermion states,

$$
\int d x \psi_{n}^{*}(x) V(x) \psi_{n^{\prime}}(x)=\pi V_{0} x_{0} \psi_{n}^{*}(0) \psi_{n^{\prime}}(0),
$$

involve the Harmonic oscillator wave-functions at $x=0$. These have the usual expression

$$
\psi_{n}(x)=\frac{x_{0}^{-1 / 2} \pi^{-1 / 4}}{2^{n / 2} n !^{1 / 2}} H_{n}\left(\frac{x}{x_{0}}\right) e^{-\frac{x^{2}}{2 x_{0}^{2}}},
$$

in terms of the Hermite polynomials $H_{n}$ with $x_{0}$ being the characteristic oscillator length. By the parity of the Hermite polynomials, $H_{n}\left(-x / x_{0}\right)=(-1)^{n} H_{n}\left(x / x_{0}\right)$, we have $H_{n}(0)=0$ for odd- $n$, i.e., $n=2 r+1$, with $r=0,1, \cdots, \infty$. Therefore, the impurity potential induces excitations which connect only unperturbed one-fermion states labelled by even
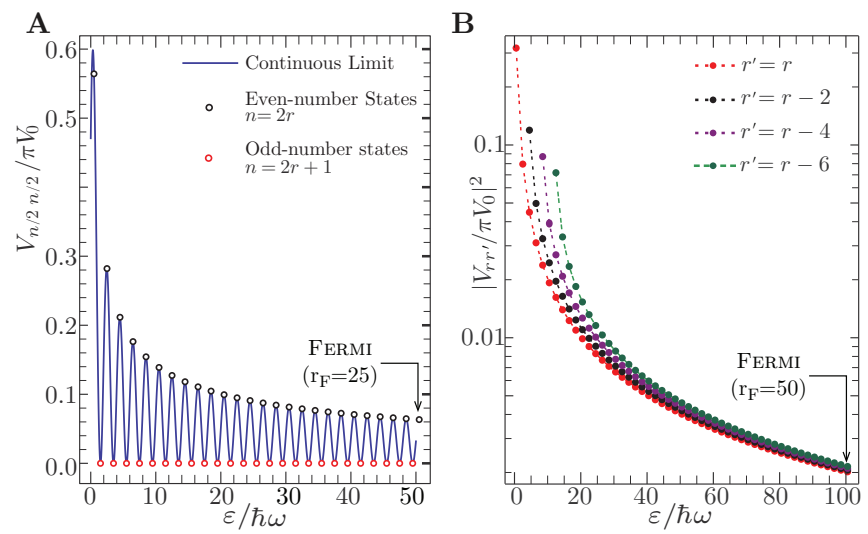

FIG. 5. (color on line) First order energy shifts for the occupied fermion levels $\varepsilon_{n}$ of the trap (A) and second order couplings between even one-fermion states (B). The continuous limit representation of $V_{n / 2 n / 2}$ in panel $\mathbf{A}$ is obtained by letting $n$ take any real value between 0 and $2 r_{\mathrm{F}}$, which corresponds to the approximation $\varepsilon_{\mathrm{F}} / \hbar \omega \gg 1$. 
numbers $n=2 r$, with $r=0,1, \cdots, \infty$. The representation of the Hermite polynomials with even numbers in power series,

$$
H_{2 r}\left(\frac{x}{x_{0}}\right)=\sum_{k=0}^{r} \frac{4^{k}(2 r) !(-1)^{r-k}}{(2 k) !(r-k) !}\left(\frac{x}{x_{0}}\right)^{2 k},
$$

allow us to write $H_{2 r}(0)=(-1)^{r} \frac{(2 r) !}{r !}$, which leads to the matrix elements

$$
\begin{aligned}
V_{r r^{\prime}} & =\int d x \psi_{2 r}^{*}(x) V(x) \psi_{2 r^{\prime}}(x) \\
& =\sqrt{\pi} V_{0} \frac{(-1)^{r+r^{\prime}}}{2^{r+r^{\prime}}} \frac{(2 r) !^{1 / 2}}{r !} \frac{\left(2 r^{\prime}\right) !^{1 / 2}}{r^{\prime} !},
\end{aligned}
$$

appearing in the second quantized representation of the impurity potential in the harmonic oscillator basis. Using the identity

$$
\gamma_{r}=\frac{\Gamma(r+1 / 2)}{\Gamma(r+1)}=2^{-2 r} \pi^{1 / 2} \frac{(2 r) !}{r !^{2}},
$$

where $\Gamma$ is the Euler gamma function, we get $V_{r r^{\prime}}=$ $V_{0}(-1)^{r+r^{\prime}} \gamma_{r}^{1 / 2} \gamma_{r^{\prime}}^{1 / 2}$. The diagonal matrix elements $V_{r r}=$ $V_{0} \gamma_{r}$, shown in Fig. 5A, entirely determine the one-vertex graph of Eq. (4), representing first order corrections to the single-particle energies $\varepsilon_{2 r}=\hbar \omega(2 r+1 / 2)$. On the other hand, both diagonal and off-diagonal matrix elements of $V(x)$, shown in Fig. 5B, appear as absolute squares, $\left|V_{r r^{\prime}}\right|^{2}=$ $V_{0}^{2} \gamma_{r} \gamma_{r^{\prime}}$, in the two-vertex graph given in Eq. (5).

\section{Appendix B: Linked Cluster expansion of the vacuum persistence amplitude}

The vacuum persistence amplitude, introduced in Eqs. (1) and (2), may be expanded by the Dyson-Wick series

$$
\nu_{\beta}(t)=1+\sum_{m=1}^{\infty} \nu_{\beta}^{(m)}(t)
$$

whose coefficients account for processes where the gas retrieves its equilibrium unperturbed configuration after $m=$ $1,2, \cdots$ 'scatterings' with the impurity potential:

$$
\nu_{\beta}^{(m)}(t)=\frac{(-i)^{m}}{\hbar^{m} m !} \int_{0}^{t} d t_{1} \cdots \int_{0}^{t} d t_{m}\left\langle T \tilde{V}\left(t_{1}\right) \cdots \tilde{V}\left(t_{m}\right)\right\rangle .
$$

In the interaction picture, with

$$
\tilde{V}(t)=\sum_{r, r^{\prime}, \xi} V_{r r^{\prime}} \hat{c}_{2 r \xi}^{\dagger}(t) \hat{c}_{2 r^{\prime} \xi}(t)
$$

the time-evolution of creation and annihilation operators is that of the undisturbed Harmonic oscillator, i.e.,

$$
\hat{c}_{2 r \xi}^{\dagger}(t)=e^{i \omega\left(2 r+\frac{1}{2}\right) t} \hat{c}_{2 r \xi}^{\dagger}, \quad \hat{c}_{2 r \xi}(t)=e^{-i \omega\left(2 r+\frac{1}{2}\right) t} \hat{c}_{2 r \xi} .
$$

Accordingly,

$$
\begin{aligned}
& \left\langle T \tilde{V}\left(t_{1}\right) \cdots \tilde{V}\left(t_{m}\right)\right\rangle=\sum_{r_{1}, r_{1}^{\prime}, \xi_{1}} V_{r_{1} r_{1}^{\prime}} \cdots \sum_{r_{m}, r_{m}^{\prime}, \xi_{m}} V_{r_{m} r_{m}^{\prime}} \\
& \quad \times\left\langle T \hat{c}_{2 r_{1} \xi_{1}}^{\dagger}\left(t_{1}\right) \hat{c}_{2 r_{1}^{\prime} \xi_{1}}\left(t_{1}\right) \cdots \hat{c}_{2 r_{m} \xi_{m}}^{\dagger}\left(t_{m}\right) \hat{c}_{2 r_{m}^{\prime} \xi_{m}}\left(t_{m}\right)\right\rangle .
\end{aligned}
$$

Here, the time-ordered average at the right-hand side may be decomposed using the Wick's theorem into sums of products where each factor is a contracted pairs of creation/anihilation operators. The central approximation of the work is to retain terms that contain only equal time and two-time contractions, namely

$$
\left\langle\cdots \hat{c}_{2 r_{j} \xi_{j}}^{\dagger}\left(t_{j}\right) \hat{c}_{2 r_{j}^{\prime} \xi_{j}}\left(t_{j}\right) \cdots\right\rangle=\cdots f_{r_{j}}^{+} \delta_{r_{j} r_{j}^{\prime}} \cdots
$$

and

$$
\begin{aligned}
& \left\langle\cdots \hat{c}_{2 r_{j} \xi_{j}}^{\dagger}\left(t_{j}\right) \hat{c}_{2 r_{j}^{\prime} \xi_{j}}\left(t_{j}\right) \cdots \hat{c}_{2 r_{i} \xi_{i}}^{\dagger}\left(t_{i}\right) \hat{c}_{2 r_{i}^{\prime} \xi_{i}}\left(t_{i}\right) \cdots\right\rangle \\
& \quad=-\hbar^{2} \cdots \delta_{\xi_{i} \xi_{j}} G_{r_{j}}^{\beta}\left(t_{i}-t_{j}\right) \delta_{r_{j} r_{i}^{\prime}} G_{r_{i}}^{\beta}\left(t_{j}-t_{i}\right) \delta_{r_{i} r_{j}^{\prime}} \cdots,
\end{aligned}
$$

where the Fermion occupation numbers $f_{r}^{+}$and the unperturbed Fermion propagators $G_{r}^{\beta}(t)$ have been introduced in the main text (see Eq. (3)). When these expressions are summed over even level numbers and integrated over time variables, we are left with products including either

$$
\Lambda_{1}^{\beta}(t)=-\frac{i(2 s+1) t}{\hbar} \sum_{r} V_{r r} f_{r}^{+}
$$

or

$$
\begin{aligned}
\Lambda_{2}^{\beta}(t)=- & \frac{(2 s+1)}{2} \sum_{r^{\prime}, r^{\prime \prime}}\left|V_{r^{\prime} r^{\prime \prime}}\right|^{2} \\
& \times \int_{0}^{t} d t^{\prime} \int_{0}^{t} d t^{\prime \prime} G_{r^{\prime}}^{\beta}\left(t^{\prime}-t^{\prime \prime}\right) G_{r^{\prime \prime}}^{\beta}\left(t^{\prime \prime}-t^{\prime}\right),
\end{aligned}
$$

which are just the connected diagrams reported in Eqs. (4) and (5). Each product equals $\Lambda_{1}^{\beta}(t)^{j} \Lambda_{2}^{\beta}(t)^{m-j}$, obtained by $\left(\begin{array}{c}m \\ j\end{array}\right)$ distinct contractions for some $j$ between 0 and $m$.

This means that

$$
\nu_{\beta}^{(m)}(t) \approx \frac{1}{m !} \sum_{j=0}^{m}\left(\begin{array}{c}
m \\
j
\end{array}\right) \Lambda_{1}^{\beta}(t)^{j} \Lambda_{2}^{\beta}(t)^{m-j} .
$$

so that the Dyson-Wick series for the vacuum persistence amplitude takes the exponential form

$$
\nu_{\beta}(t)=\sum_{m=0}^{\infty} \frac{\left[\Lambda_{1}^{\beta}(t)+\Lambda_{2}^{\beta}(t)\right]^{m}}{m !}=e^{\Lambda_{1}^{\beta}(t)+\Lambda_{2}^{\beta}(t)} .
$$

The single vertex graph (4) gives rise to the first order energy shift discussed in the main text, while the two-vertex connected graph has a more involved structure. Performing the 
time-ordered integrals in (5) we rewrite it as

$$
\Lambda_{2}^{\beta}(t)=-\frac{\alpha \varepsilon_{\mathrm{F}}}{\hbar} \sum_{r, r^{\prime}=0}^{\infty}\left[i t \varphi_{r r^{\prime}}+\frac{\psi_{r r^{\prime}}(t)}{2 \omega}\right] f_{r}^{+} f_{r^{\prime}}^{-},
$$

in which

$$
\varphi_{r r^{\prime}}=\frac{\gamma_{r} \gamma_{r^{\prime}}}{r-r^{\prime}}, \quad \psi_{r r^{\prime}}(t)=\varphi_{r r^{\prime}} \frac{1-e^{2 i\left(r-r^{\prime}\right) t \omega}}{r-r^{\prime}} .
$$

Here, we may separate the sums over even-state labels $\left(r, r^{\prime}\right)$, so that the two-vertex connected graph can then be split into three contributions:

$$
\Lambda_{2}^{\beta}(t)=\Lambda_{2 \mathrm{~S}}^{\beta}(t)+\Lambda_{2 \mathrm{G}}^{\beta}(t)+\Lambda_{2 \mathrm{P}}^{\beta}(t),
$$

where the subscripts stand for Shift, Gaussian and Periodic, respectively. In particular:

(i) the off-diagonal summands in (B1) that multiply $\varphi_{r r^{\prime}}$ give rise to $\Lambda_{2 \mathrm{~s}}^{\beta}(t)=-i t E_{2}^{\beta} / \hbar$, where

$$
E_{2}^{\beta}=\alpha \varepsilon_{\mathrm{F}} \sum_{r \neq r^{\prime}=0}^{\infty} f_{r}^{+} \varphi_{r r^{\prime}} f_{r^{\prime}}^{-}
$$

is the energy correction reported in Eq. (7)

(ii) the diagonal elements of Eq. (B1) yield the quadratic power law in $\Lambda_{2 G}^{\beta}(t)$ [see Eq. (8)];

(iii) the remaining terms of the series in Eq. (B1) give the time periodic sub-diagram

$$
\Lambda_{2 \mathrm{P}}^{\beta}(t)=-\frac{\alpha \varepsilon_{\mathrm{F}}}{2 \hbar \omega} \sum_{r \neq r^{\prime}}^{\infty} f_{r}^{+} \psi_{r r^{\prime}} f_{r^{\prime}}^{-}
$$

also reported in Eq. (9).

\section{Appendix C: Numerical computations}

As shown in the main text, the real and imaginary parts of the connected graphs $\Lambda_{1}^{\beta}(t)$ [Eqs. (4), Fig. 1B] and $\Lambda_{2}^{\beta}(t)$ [Eq. (5), Fig. 1C, Fig. 2] combine in the vacuum persistence amplitude to give:

$$
\nu_{\beta}(t)=e^{-\frac{i t}{\hbar}\left(E_{1}^{\beta}+E_{2}^{\beta}\right)} e^{-\alpha g_{\beta} \omega^{2} t^{2}} e^{\Lambda_{2 \mathrm{p}}^{\beta}(t)} .
$$

The knowledge of $\nu_{\beta}(t)$, allows us to determine the decoherence factor $\left|\nu_{\beta}(t)\right|$ (Fig. $\left.3 \mathbf{A}, \mathbf{C}, \mathbf{E}\right)$, the shake up spectrum $\left|\tilde{\nu}_{\beta}(E)\right|$ (Fig. 3B, D, F, Fig 4A), and the non-Markovianity measure $\mathcal{N}$ [Eq. (15), Fig 4B]. Then, the basic quantities in our calculations are:

(i) the first and second order corrections, $E_{1}^{\beta}$ [Eq. (6), Fig. 1B] and $E_{2}^{\beta}$ [Eqs. (7), Fig. 1C], to the equilibrium energy $E_{0}^{\beta}$ (Fig. 1A); (ii) the coefficient $g_{\beta}$ determining the standard deviation $\delta_{\beta}$ [Eq. (8), Fig. 2A] of the Gaussian sub-diagram $\Lambda_{2 \mathrm{G}}^{\beta}$;

(iii) the shake-up sub-diagram $\Lambda_{2 \mathrm{P}}^{\beta}$ [Eqs. (9), Fig. 2B].

These contributions contain summations running over all one-fermion eigenstates of the trap weighted by the fermi factors $f_{r}^{+}, f_{r^{\prime}}^{-}$. These are expressed as $f_{r}^{+}=[1+$ $\left.e^{2 \beta \hbar \omega\left(r-r_{\mu}\right)}\right]^{-1}$ and $f_{r^{\prime}}^{-}=\left[1+e^{-2 \beta \hbar \omega\left(r-r_{\mu}\right)}\right]^{-1}$, using the parametrization $\mu=\hbar \omega\left(2 r_{\mu}+1 / 2\right)$ for the chemical potential. The index $r_{\mu}$ tends to $r_{\mathrm{F}}+1 / 4$ for $\beta \rightarrow \infty$, so that the $\mu$ lies in the middle between the highest occupied $\left(\varepsilon_{\mathrm{F}}=\varepsilon_{2 r_{\mathrm{F}}}\right)$ and the lowest unoccupied one-fermion levels $\left(\varepsilon_{2 r_{\mathrm{F}}+1}\right)$ of the gas. For finite $\beta$ we determined $r_{\mu}$ by numerically constraining the conservation of particle number:

$$
2 r_{\mathrm{F}}+1=\sum_{r}\left(f_{r}^{+}+f_{r+1 / 2}^{+}\right)
$$

As shown in Fig. 6, $r_{\mu}$ and hence $\mu$, reach their maximum values at the absolute zero $(\beta \rightarrow \infty)$. They decrease with decreasing $\beta$ and take largely negative values for $\beta \rightarrow 0$ where the classical limit applies. Interestingly enough, both $r_{\mu}$ and $\mu$ are almost independent on temperature for $\beta \hbar \omega \gtrsim 0.4$ and $r_{\mathrm{F}}=5-500$.
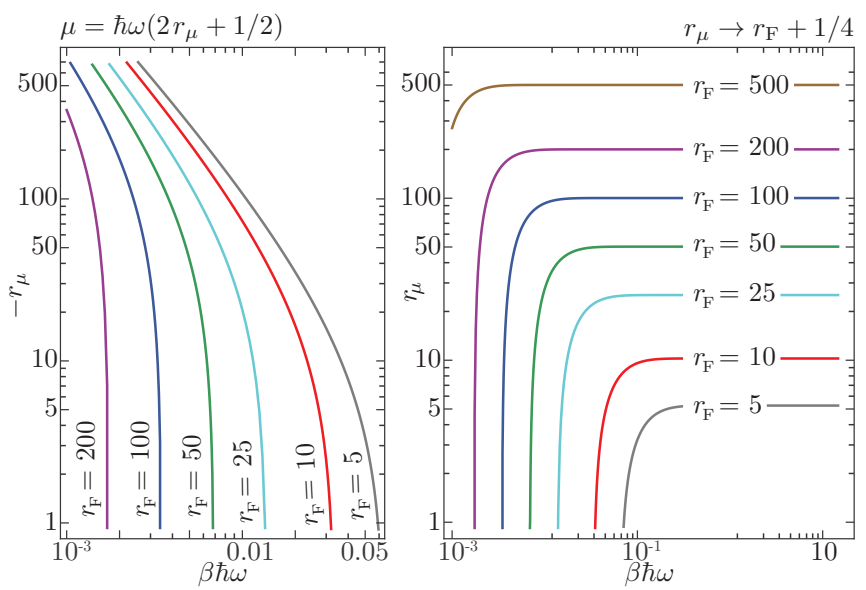

FIG. 6. Chemical potential index $r_{\mu}$ vs $\beta \hbar \omega$, for $r_{\mathrm{F}}=5-500$

With the computed $f_{r}^{ \pm}$-distributions, we run numerical computations of the basic quantities (i)-(iii) using a high energy cut-off $\varepsilon_{\text {CUT }} \gg \varepsilon_{\mathrm{F}}$. To fix $\varepsilon_{\text {CUT }}=\hbar \omega\left(2 r_{\text {CUT }}+1 / 2\right)$, we performed convergency tests by changing $r_{\mathrm{CUT}}$ in order to have a maximum instability error below $0.1 \%$.

In our applications to a spin $1 / 2$ gas, we observed that accurate estimations of $E_{2}^{\beta}$ and $g_{\beta}$ require values of $r_{\text {CUT }}$ of the order of $10^{4}$ for $r_{\mathrm{F}}$ below $\sim 200$ and $\beta \hbar \omega$ larger than $\sim 10^{-5}$. In particular, the plots of Fig. $1 \mathbf{C}$ and Fig. $2 \mathbf{A}$ of the main text were generated with a energy cut-off of $10-10^{3} \varepsilon_{\mathrm{F}}$. As for $E_{0}^{\beta}, E_{1}^{\beta}$, and $\Lambda_{2 \mathrm{P}}^{\beta}$, we found out a cut-off of $\sim 10 \varepsilon_{\mathrm{F}}$ to be sufficient in the investigated ranges of fermion numbers and temperatures. Accordingly, we included up to $10^{3}$ one-fermion states in Fig. 1A-B and Fig. $2 \mathbf{C}$ of the main text. 
To provide a more complete picture of the basic quantities involved in the numerical calculations, in Fig. $7 \mathbf{A}-\mathbf{C}$ we report the behavior of the equilibrium energy and the two energy shifts $v s \beta \hbar \omega$ for gases made of different fermion numbers. We remark that both $E_{1}^{\beta}$ and $E_{2}^{\beta}$ are indeed small corrections to the unperturbed value $E_{0}^{\beta}$ of a spin- $1 / 2$ gas, for values of the critical exponent $\alpha \lesssim 1$. In addition, $E_{1}^{\beta}$ is generally larger that $E_{2}^{\beta}$. These energies are strongly affected by the number of particles in the gas and weakly dependent on temperature for $\beta \hbar \omega \gtrsim 0.4$.

On the other hand the Gaussian damping/broadening brought by $\Lambda_{2 \mathrm{G}}^{\beta}$, with standard deviation $\delta_{\beta}$, is almost entirely dependent on the thermal energy $\beta \hbar \omega$ and the critical exponent $\alpha$ (Fig. 7D). This contribution leads to a smearing of the shake up response of the system in way that resembles the Anderson-Yuval approach to the Kondo problem [2]. Indeed, $\delta_{\beta}$ decreases exponentially to zero with increasing $\beta \hbar \omega$, following the limiting trend

$$
\delta_{\beta} \approx 2^{3 / 2} \alpha^{1 / 2} e^{-\beta \omega \hbar / 4}
$$

for $\beta \hbar \omega \gtrsim 7-8$ (Figs. $2 \mathbf{A}$ and $7 \mathbf{B}$ ).
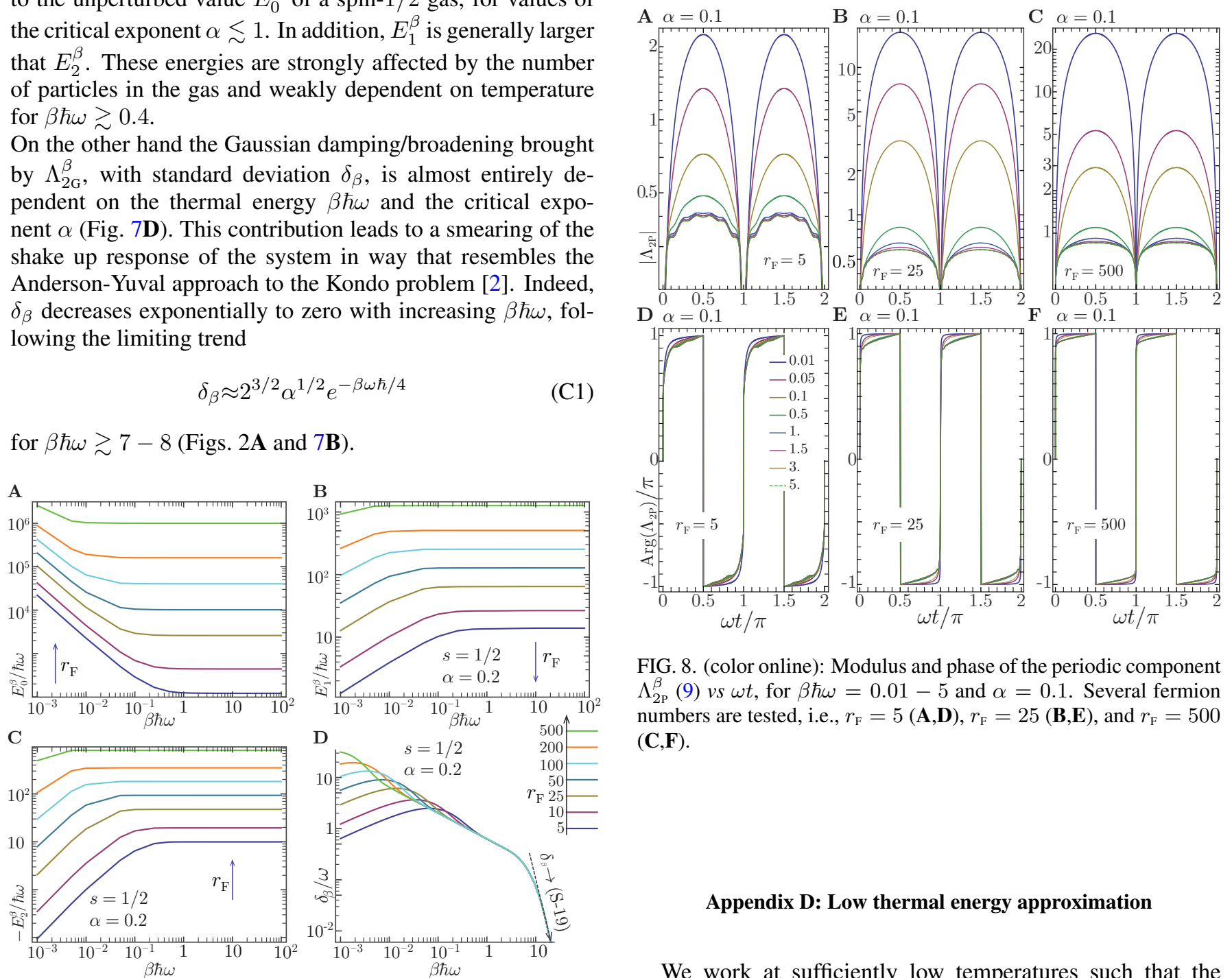

FIG. 8. (color online): Modulus and phase of the periodic component $\Lambda_{2 \mathrm{P}}^{\beta}$ (9) $v s \omega t$, for $\beta \hbar \omega=0.01-5$ and $\alpha=0.1$. Several fermion numbers are tested, i.e., $r_{\mathrm{F}}=5(\mathbf{A}, \mathbf{D}), r_{\mathrm{F}}=25(\mathbf{B}, \mathbf{E})$, and $r_{\mathrm{F}}=500$ $(\mathbf{C}, \mathbf{F})$.

\section{Appendix D: Low thermal energy approximation}

We work at sufficiently low temperatures such that the chemical potential is well approximated by its maximum value

$$
\mu \underset{\beta \hbar \omega \rightarrow \infty}{\rightarrow} \hbar \omega\left(2 r_{\mathrm{F}}+1\right)
$$

Fig. 1 of the main text). All Energy values are expressed in units of $\hbar \omega$ vs $\beta \hbar \omega$ for different Fermi numbers/energies and temperatures. Significant changes are induced by $r_{\mathrm{F}}$, whereas negligible differences are observed in the energy curves for $\beta \hbar \omega>0.4$. Gaussian standard deviation $\delta_{\beta}$ (panel D) appearing in the two-vertex contribution $\Lambda_{2 \mathrm{G}}^{\beta}$ and reported in units of $\omega$ for the same values of $r_{\mathrm{F}}$ and $\alpha$ used in the plots of panels (A-C).

In addition, as evident by comparing Fig. $2 \mathbf{B}$ of the main text with the plots of Fig. 8, the sub-diagram $\Lambda_{2 \mathrm{P}}^{\beta}$ has a time period of $\pi / \omega$. Its modulus $\left|\Lambda_{2 \mathrm{P}}^{\beta}\right|$ presents zeroes at $\omega t=m \pi$ and maxima at $\omega t=m \pi / 2$, with $m=0, \pm 1, \pm 2, \cdots$. The intensities of such maxima (Fig. 8A-C) increase with increas-

ing the Fermi number $\left(2 r_{\mathrm{F}}\right)$, the critical exponent $(\alpha)$, and the thermal energy $\left(\beta^{-1}\right)$. The phase of $\Lambda_{2 \mathrm{P}}^{\beta}$ is discontinuous at the extremes of $\left|\Lambda_{2 \mathrm{P}}^{\beta}\right|$ and less dependent on these parameters (Fig. 8D-F).

FIG. 7. (color on line) Equilibrium energy $E_{\beta}^{0}$ of a spin- $1 / 2$ gas into a harmonic trap (panel $\mathbf{A}$ ) compared with first and second order corrections, $E_{\beta}^{1}$ [Eq. (6), panel B] and $E_{\beta}^{2}$ [Eq. (7), panel C], due value

corresponding to

$$
r_{\mu} \underset{\beta \hbar \omega \rightarrow \infty}{\rightarrow} r_{\mathrm{F}}+1 / 4,
$$

as shown in Fig. 6. Then, we consider systems with a relatively large number of particles $\left(r_{\mathrm{F}} \gtrsim 10\right)$ and focus on the sub-diagrams $\Lambda_{2 \mathrm{G}}^{\beta}(t)$ and $\Lambda_{2 \mathrm{P}}^{\beta}(t)$. In this, way we provide the formal justifications for the analytical approximations introduced in Eqs. (11) and (12), which determine the 'unshifted' amplitude $\nu_{\beta}^{\prime}(t)$ given in Eq. (10) and the excitation spectrum $\tilde{\nu}_{\beta}^{\prime}(E)$, shown in Fig $4 \mathbf{A}$. 
Using the power series

$$
f_{r}^{+} f_{r}^{-} \underset{\varepsilon_{2 r} \lessgtr \mu}{=} \sum_{m=1}^{\infty}(-1)^{m+1} m e^{ \pm \beta m\left(\varepsilon_{2 r}-\mu\right)},
$$

we rewrite the Gaussian damping parameter, reported in Eq. (8), as

$$
g_{\beta}=\sum_{m=1}^{\infty}(-1)^{m+1} m g_{m}^{\beta}
$$

The coefficients of this expansion read

$$
\begin{aligned}
g_{m}^{\beta} & =\frac{\varepsilon_{\mathrm{F}}}{\hbar \omega} \sum_{r<r_{\mu}} \gamma_{r}^{2} e^{\beta m\left(\varepsilon_{2 r}-\mu\right)}+\frac{\varepsilon_{\mathrm{F}}}{\hbar \omega} \sum_{r>r_{\mu}} \gamma_{r}^{2} e^{-\beta m\left(\varepsilon_{2 r}-\mu\right)} \\
& =\frac{\varepsilon_{\mathrm{F}}}{\hbar \omega} \sum_{r<r_{\mu}} \gamma_{r}^{2} e^{-2 \omega \tau_{m}\left(r_{\mu}-r\right)}+\frac{\varepsilon_{\mathrm{F}}}{\hbar \omega} \sum_{r>r_{\mu}} \gamma_{r}^{2} e^{-2 \omega \tau_{m}\left(r-r_{\mu}\right)},
\end{aligned}
$$

with $\tau_{m}$ being the characteristic times $\tau_{m}=m \beta \hbar$, induced by thermal fluctuations. Then, we use Eq. (D2) and perform a change of summation indices to write

$$
\begin{aligned}
g_{m}^{\beta}= & \frac{\varepsilon_{\mathrm{F}}}{\hbar \omega} e^{-2 \omega \tau_{m}\left(r_{\mu}-r_{\mathrm{F}}\right)} \sum_{r=0}^{r_{\mathrm{F}}} \gamma_{r_{\mathrm{F}}-r}^{2} e^{-2 \omega \tau_{m} r} \\
& +\frac{\varepsilon_{\mathrm{F}}}{\hbar \omega} e^{2 \omega \tau_{m}\left(r_{\mu}-r_{\mathrm{F}}\right)} \sum_{r=1}^{\infty} \gamma_{r_{\mathrm{F}}+r}^{2} e^{-2 \omega \tau_{m} r} .
\end{aligned}
$$

The transformed summations in this last line are dominated by low $r$ terms. In a many fermion environment, we may use the asymptotic relation $\gamma_{r_{\mathrm{F}} \pm r}^{2} \approx \gamma_{r_{\mathrm{F}}}^{2} \approx r_{\mathrm{F}}^{-1}$ and obtain

$$
g_{m}^{\beta} \underset{r_{\mathrm{F}} \gg 1}{\approx} 2 \frac{e^{2 \omega \tau_{m}\left(r_{\mu}-r_{\mathrm{F}}\right)}+e^{2 \omega \tau_{m}\left(r_{\mathrm{F}}-r_{\mu}+1\right)}-e^{-2 \omega \tau_{m} r_{\mu}}}{e^{2 \omega \tau_{m}}-1} .
$$

Then, using the asymptotic form (D2) and neglecting $e^{-2 \omega \tau_{m} r_{\mu}}$, we find

$$
g_{m}^{\beta} \approx 2 \frac{e^{\omega \tau_{m} / 2}}{e^{\omega \tau_{m}}-1}
$$

which leads to Eq. (11), i.e.,

$$
g_{\beta} \approx 2 \sum_{m=1}^{\infty}(-1)^{m} m \frac{e^{\omega \tau_{m} / 2}}{e^{\omega \tau_{m}}-1},
$$

and let us approximate the standard deviation with

$$
\delta_{\beta} \approx 2 \alpha^{1 / 2}\left[\sum_{m=1}^{m_{\text {CUT }}}(-1)^{m} m \frac{e^{\omega \tau_{m} / 2}}{e^{\omega \tau_{m}}-1}\right]^{1 / 2} .
$$

Eq. (D3), plotted in Fig. 9A for $m_{\text {CUT }}=1,100$, is independent of the number of particles in the gas. We have verified that the truncated series for $m_{\text {CUT }}=100$ works extremely well for $r_{\mathrm{F}}=5-500$ and $\beta \hbar \omega \gtrsim 0.4$. The asymptotic form of $\delta_{\beta}$ leads to the result reported in Fig2A of the main text.

As for the Fermi-edge component $\Lambda_{2 \mathrm{P}}^{\beta}(t)$, we consider the

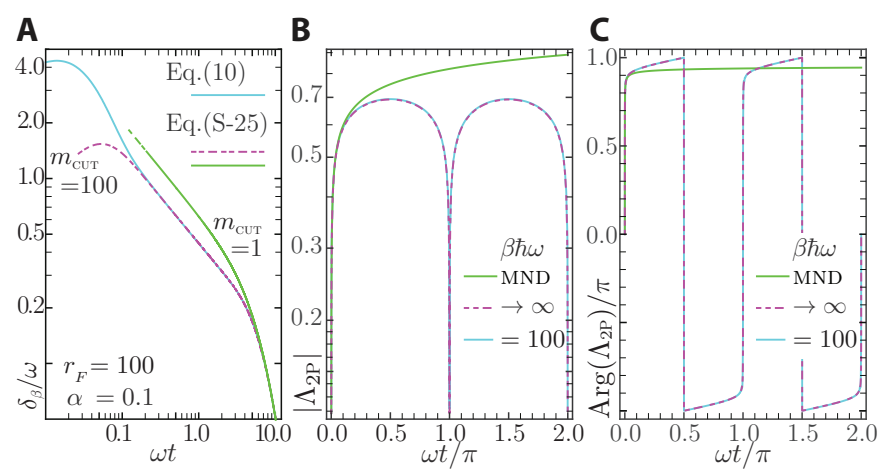

FIG. 9. (color online) (A) Standard deviation $\delta_{\beta}$ of the Gaussian diagram $\Lambda_{2 \mathrm{G}}^{\beta}(t) ;(\mathbf{B}, \mathbf{C})$ absolute value and phase of the Fermi edge diagram $\Lambda_{2 \mathrm{P}}^{\beta}(t)$, for $\alpha=0.1$ and $r_{\mathrm{F}}=100$. In panel $\mathbf{A}$, numerical computations from Eq. (8) are compared with the truncated series (D3) for $m_{\mathrm{CUT}}=1,100$. In panels $\mathbf{B}, \mathbf{C}$, numerical computations of $\Lambda_{2 \mathrm{P}}^{\beta}$, for $\beta \hbar \omega=100$, are shown together with the analytical approximation obtained from Eq. (D8), in which $\omega \tau_{0}=0.001$, and the results form the MND theory [see Eq. (14)].

auxiliary functions $\lambda_{ \pm}^{\beta}(t)$ introduced in the main text, which enter the connected graph $\Lambda_{2}^{\beta}(t)$ (see Eq. (5)). We replace the particle-hole distributions $f_{r}^{ \pm}$with the power series expansion

$$
\begin{aligned}
f_{r}^{ \pm} & =\sum_{m=0}^{\infty}(-1)^{m} e^{ \pm \beta m\left(\varepsilon_{2 r}-\mu\right)} & & \varepsilon_{2 r} \lessgtr \mu \\
& =-\sum_{m=1}^{\infty}(-1)^{m} e^{\mp \beta m\left(\varepsilon_{2 r}-\mu\right)} & & \varepsilon_{2 r} \gtrless \mu
\end{aligned}
$$

to write

$$
\lambda_{ \pm}^{\beta}(t)=\sum_{m=0}^{\infty}(-1)^{m} \lambda_{m \pm}^{\beta}(t) .
$$

Here, the coefficients $\lambda_{m \pm}^{\beta}(t)$ may be computed exactly by the finite summations

$$
\sum_{r=r_{1}}^{r_{2}} \gamma_{r} z^{r}=z^{r_{1}}{ }_{2} \tilde{F}_{1}\left(r_{1}, z\right)-z^{r_{2}+1}{ }_{2} \tilde{F}_{1}\left(r_{2}+1, z\right),
$$

holding for any $z \neq 1$, in which ${ }_{2} \tilde{F}_{1}$ is the regularized Hypergeometric function

${ }_{2} \tilde{F}_{1}\left(r_{\mathrm{F}}, z\right)=\gamma_{r_{\mathrm{F}} 2} F_{1}\left(1,1 / 2+r_{\mathrm{F}}, 1+r_{\mathrm{F}} ; z\right)$

Working in the temperature range where Eq. (D1) holds, the $m=0$ coefficients of the series (D4) turn out to be independent on $\beta$ :

$$
\begin{aligned}
\lambda_{0+}^{\beta}(t)= & \frac{\sqrt{\pi}}{\sqrt{1-e^{2 i t \omega}}} \\
& -e^{\frac{i t \varepsilon_{\mathrm{F}}}{\hbar}+\frac{3 \omega i t}{2}}{ }_{2} \tilde{F}_{1}\left(r_{\mathrm{F}}+1, e^{2 i t \omega}\right), \\
\lambda_{0-}^{\beta}(t)= & e^{-\frac{i t \varepsilon_{\mathrm{F}}}{\hbar}-\frac{3 \omega i t}{2}}{ }_{2} \tilde{F}_{1}\left(r_{\mathrm{F}}+1, e^{-2 i t \omega}\right) .
\end{aligned}
$$


The other coefficients, accounting for low temperature effects, have the form:

$$
\begin{aligned}
\lambda_{m \pm}^{\beta}(t)= & \pm \frac{\sqrt{\pi} e^{-\beta m \varepsilon_{\mathrm{F}}}}{\sqrt{1-e^{2 \omega\left(\tau_{m} \pm i t\right)}}} \\
& \mp e^{ \pm \frac{i t \varepsilon_{\mathrm{F}}}{\hbar} \pm \frac{3}{2} \omega\left(i t+\tau_{m}\right)}{ }_{2} \tilde{F}_{1}\left[r_{\mathrm{F}}+1, e^{2 \omega\left(\tau_{m} \pm i t\right)}\right] \\
& \mp e^{ \pm \frac{i t \varepsilon_{\mathrm{F}}}{\hbar} \pm \frac{3}{2} \omega\left(i t-\tau_{m}\right)}{ }_{2} \tilde{F}_{1}\left[r_{\mathrm{F}}+1, e^{2 \omega\left( \pm i t-\tau_{m}\right)}\right],
\end{aligned}
$$

With the zero temperature parts (D7) and (D6), we need to add an imaginary time regularization to the $t^{\prime \prime}$-integral in the twoloop term (5), i.e., we have to shift the $t^{\prime \prime}$ integration domain by $i \tau_{0}$ to prevent ${ }_{2} \tilde{F}_{1}\left(r_{\mathrm{F}}+1, e^{ \pm 2 \omega r i t^{\prime \prime}}\right)$ from being singular. We may, then, insert Eq. (D4) in the expression for $\Lambda_{2}^{\beta}(t)$, compute the $r$-summations, and use the large $r_{\mathrm{F}}$ expansion

$$
{ }_{2} \tilde{F}_{1}\left(r_{\mathrm{F}} \gg 1, z\right)=\frac{r_{\mathrm{F}}^{-1 / 2}}{1-z}+\mathrm{o}\left(r_{\mathrm{F}}^{-3 / 2}\right) \text {. }
$$

Finally, we may perform the $t^{\prime}$ and $t^{\prime \prime}$-integrals, excluding terms proportional to $t$ and $t^{2}$. What is left is a combination of logarithmic and polylogarithmic functions, dominated by the Fermi-edge term reported in Eq. (12). Indeed, as shown in Fig. 9, the zero temperature form

$$
\Lambda_{2 \mathrm{P}}^{\infty}(t) \approx \ln \left[\frac{e^{2 \tau_{0} \omega}-1}{e^{2 \omega\left(\tau_{0}+i t\right)}-1}\right]^{\alpha}
$$

obtained from this procedure is in excellent agreement with the numerical calculations reported in Fig. $2 \mathbf{B}$ for $r_{\mathrm{F}}=100$ and $\beta \hbar \omega$ larger than $\sim 1$. The reliability of such an approximation is also attested by the comparison in Fig $4 \mathbf{A}$. We will see in the following appendix that Eq. (D8) provides an accurate description of the singular response of a Fermi gas with low numbers of particles.

In the main text, we observed that $\Lambda_{2 \mathrm{P}}^{\infty}(t)$ correctly tends to the MND form given by Eq. (14) when the harmonic trap frequency is lowered to zero, keeping the number of particles in the gas finite. The singularity index is proportional to the height of the impurity potential barrier. Nozières and De Dominicis [4] calculated the propagator for a free electron in the transient potential activated by a structure-less corehole, by solving the associated Dyson equation in the longtime limit with Muskhelishvili techniques for singular integral equations. They assumed a constant potential of arbitrary height and width $\hbar / \tau_{0}$ around the Fermi level of the gas and found the singularity index to depend of the phase-shifts of this potential. Therefore they provided an asymptotic expression for all closed loops $\Lambda^{\infty}(t)=\sum_{n} \Lambda_{n}^{\infty}(t)$, which may be written as Eq. (14). In our derivation, we have given a model to the impurity potential, being described by non constant matrix elements $V_{r r^{\prime}}$ (see appendix A). In addition we have found an analytical form for two vertex graph, Eq. (12), which accurately describes $\Lambda_{2 \mathrm{P}}^{\beta}(t)$ at any time $t$ for a sufficiently wide range of temperatures and particle number. We expect the non trivial part of the higher order contributions $\Lambda_{n>2}^{\beta}(t)$ to change the value of the $\alpha$-parameter in such a way that it will depend on the phase shifts of the impurity potential.

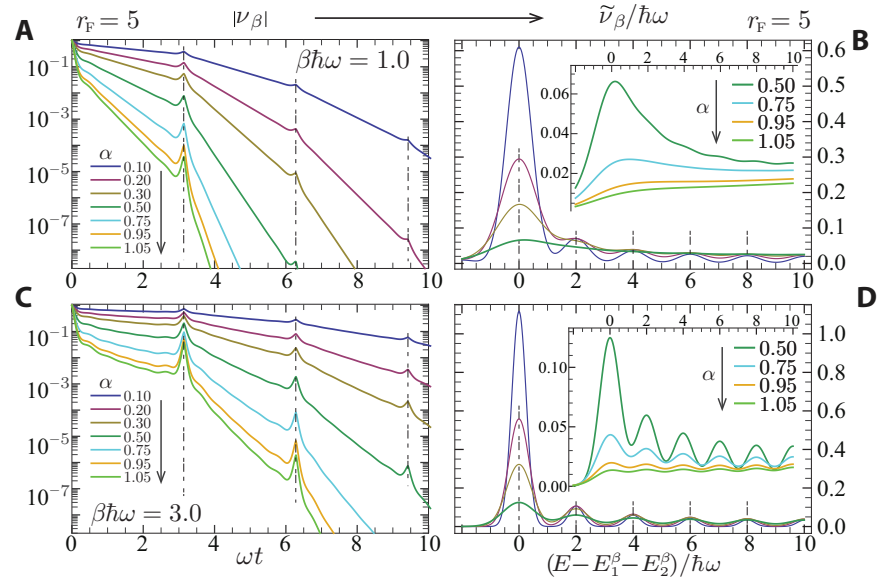

FIG. 10. (color online): Absolute value of the decoherence factor $\left|\nu_{\beta}(t)\right|$ (left panels, $\mathbf{A}, \mathbf{C}$ ) and excitation spectrum $\tilde{\nu}_{\beta}^{\prime}(E)$ (right panels, B,D), calculated from Eq. (10) by numerically computing the Gaussian damping (8) and the periodic sub-diagram (9), for $\beta \hbar \omega=1,3, r_{\mathrm{F}}=5$, and $\alpha=0.1-1.05$.

\section{Appendix E: Decoherence factor, excitation spectrum and Non markovianity measure}

With the arguments given in the previous section, the decoherence factor takes the analytical approximation

$$
\begin{aligned}
\left|\nu_{\beta}(t)\right|= & e^{-2 \alpha \omega^{2} t^{2} \sum_{m=1}^{\infty}(-1)^{m} m e^{-\beta \hbar \omega m / 2}} \\
& \times \prod_{m=-\infty}^{\infty}\left|\frac{e^{2 \tau_{m} \omega}-1}{e^{2\left(i t+\tau_{m}\right) \omega}-1}\right|^{\alpha}
\end{aligned}
$$

and the excitation spectrum relative to the perturbed equilibrium energy of the gas may be written

$$
\tilde{\nu}_{\beta}^{\prime}(E)=\int_{0}^{\infty} \frac{d t}{2 \pi \hbar} e^{\frac{i t}{\hbar}\left(E+E_{1}^{\beta}+E_{2}^{\beta}\right)} \nu_{\beta}^{\prime}(t) .
$$

We performed numerical calculations of both $\left|\nu_{\beta}(t)\right|$ and $\tilde{\nu}_{\beta}^{\prime}(E)$ by selecting different fermion numbers $\left(r_{\mathrm{F}}=5-\right.$ $100)$, coupling parameters $(\alpha=0.1-1.05)$, and thermal energies $(\beta \hbar \omega=0.001-10)$. Then, we evaluated the maxima/minima of $\left|\nu_{\beta}(t)\right|$ in a finite time-window with $t=0-100 \delta_{\beta}^{-1}$ and plugged the differences $\left|\nu_{\beta}\left(t_{\max , n}\right)\right|-$ $\left|\nu_{\beta}\left(t_{\min , n}\right)\right|$ into Eq. (15) to estimate the non-Markovianity $\mathcal{N}$ of the two-level impurity in the gas.

In Fig. 3 and $\mathbf{4 A}$, we have presented an application to a Fermi gas of 402 particles $\left(r_{\mathrm{F}}=100\right)$ where the Fermi-edge behavior, superimposed on a Gaussian damping trend, appears as a sequence of spikes in $\left|\nu_{\beta}(t)\right|=\left|\nu_{\beta}^{\prime}(t)\right|$ and an asymmetric peak structure in $\tilde{\nu}_{\beta}^{\prime}(E)$. Such features becoming more and more marked with decreasing temperature, which reduces the effect of the Gaussian damping $\delta_{\beta}$ (see Fig. 2A), correspond to a sharp peak of $\mathcal{N}$ at $\alpha<0.2$ (Fig. 4B). Similar considerations hold for environments containing low fermion numbers (i.e, for $r_{\mathrm{F}}=5$ in Fig. 10 and Fig. 11A) where shakeup effects are even more visible because of the decreasing 


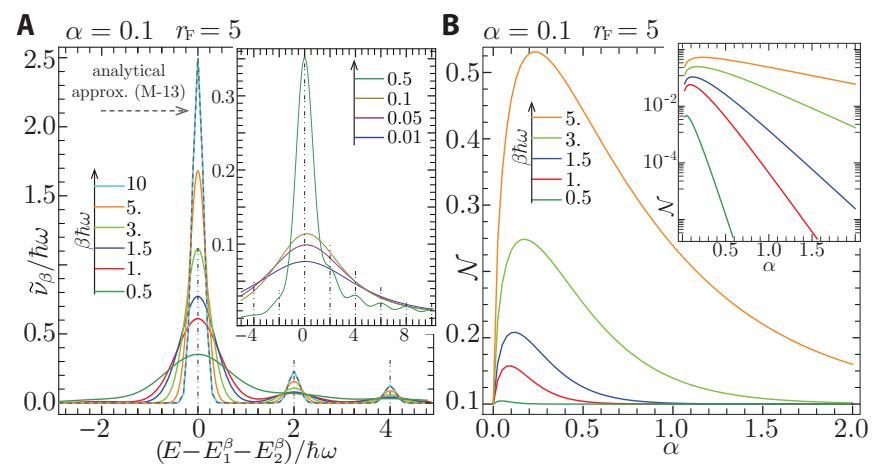

FIG. 11. (color online): (A) Absorption spectrum $\nu_{\beta}^{\prime}(E)$, calculated numerically from Eq. (10) with $\beta \hbar \omega=0.1-\infty, r_{\mathrm{F}}=5$, and $\alpha=0.1$, and analytical approximation $\nu_{\infty}^{\prime}(E)$ obtained from Eqs. (11) and (12) with $\hbar \omega \beta=10$ and $\omega \tau_{0}=0.016$; (B) NonMarkovianity measure as a function of the critical parameter $\alpha$ for various temperatures. of $\delta_{\beta}$ with decreasing $\varepsilon_{\mathrm{F}}$, leading to a sharp peaks in $\mathcal{N}$ at $\alpha<0.5$ (Fig. 11B). We finally notice that the analytical the approximation given by Eqs. (11) and (12) works extremely well with environments containing both low (Fig. 4A) and large (Fig. 11A) fermion numbers. 